where first- and second-order cords are attached to the underside of this area. In our case the rough zone was totally covered by the lesions. Operatively, the biggest part of the mass looked like an "active volcano," and the small lesions had a "lava flow" shape (Figure 2). Given this unusual and unexpected diagnosis and the young age of the patient, we opted for exclusive tumor ablation without mitral valve replacement. Although the infiltration of valvular tissue by the PFE is impossible to prove inasmuch as the mitral valve was not extracted, the natural history of this tumor is unknown and preserving the native mitral valve could have been the optimal option. To the best of our knowledge this is the first case of invading PFE on the anterior leaflet of the mitral valve reported in the literature. Clinicians should be aware of this PFE presentation to decide how to manage such patients surgically.

\title{
Total arch replacement through a midsternotomy for a right-sided aortic arch aneurysm with an aberrant left subclavian artery
}

\author{
Masashi Kai, MD, Hitoshi Okabayashi, MD, PhD, Yoshiharu Soga, MD, PhD, Michiya Hanyu, MD, PhD, \\ Takuya Nomoto, MD, PhD, Jota Nakano, MD, Hideki Kitamura, MD, Takehiko Matsuo, MD, Tomohiro Tsunekawa, MD, \\ Eitaro Umehara, MD, and Masahide Kawato, MD, Fukuoka, Japan
}

$\mathrm{T}$ he surgical approach for a right-sided aortic arch aneurysm with an aberrant left subclavian artery (ALSA) has to be considered carefully. ${ }^{1-4}$ We have successfully performed total arch replacement for a right-sided aortic arch aneurysm with an ALSA through a midsternotomy alone in 2 consecutive cases. The present article describes the surgical technique we used.

\section{Clinical Summary}

Two male patients, aged 55 and 73 years, respectively, were referred to our institution within a period of 1 month for surgical repair of a right-sided aortic arch aneurysm. Both were asymptomatic. They weighed 72 and $65 \mathrm{~kg}$. Multidimensional computed tomography showed a right-sided aortic arch with an ALSA. The aneurysm was located at the base of the ALSA (Kommerell's diverticulum). With regard to the arrangement of the arch vessels, a left common carotid artery arose from the aorta as the first branch, followed by a right subclavian artery, a right carotid artery, and the ALSA (Figure 1). No

From the Department of Cardiovascular Surgery, Kokura Memorial Hospital, Fukuoka, Japan.

Received for publication July 30, 2006; revisions received Aug 12, 2006 ; accepted for publication Aug 25, 2006.

Address for reprints: Hitoshi Okabayashi, MD, PhD, Department of Cardiovascular Surgery, Kokura Memorial Hospital, Kifunemachi 1-1, Kokurakita-ku, Kitakyusyu City, Fukuoka, 802-8555, Japan (E-mail: cardiovascsurg@siren.ocn.ne.jp).

J Thorac Cardiovasc Surg 2006;132:1473-5

$0022-5223 / \$ 32.00$

Copyright (C) 2006 by The American Association for Thoracic Surgery doi:10.1016/j.jtcvs.2006.08.033 concomitant congenital heart disease was identified by means of preoperative evaluation in either of the patients.

\section{Surgical Technique}

Through a midsternotomy, cardiopulmonary bypass was achieved with cannulation of the ascending aorta and right atrium. The first 3 arch vessels were easily exposed and taped. After initiation of core cooling, the ALSA was identified by means of palpation and divided from the left side of the anterior mediastinum (Figure 2, A and $B$ ). Circulatory arrest was established at a core temperature of $26^{\circ} \mathrm{C}$. The aortic arch was incised, and antegrade selective cerebral perfusion was established. Four balloon-tip cannulas were inserted directly into the right subclavian artery, the right common carotid artery, and the left common carotid artery from inside the aortic arch and the transected ALSA from outside the aorta. A 20-mm Hemashield quadrifurcated graft (Boston Scientific, Natick, Mass) was used for reconstruction. While dissecting the distal arch, the right phrenic nerve was identified and taped to prevent injury. The descending aorta was completely transected, and open distal anastomosis was performed in an elephant trunk manner by using a short graft (Figure 2, $C$ and $D$ ). The quadrifurcated graft was anastomosed to the short graft pulled out of the descending aorta. Reperfusion and rewarming were started through the femoral artery. Then the proximal end of the quadrifurcated graft was anastomosed to the aortic root, and the aorta was declamped. Finally, the arch vessels were independently reconstructed by using, in order, the branches of the graft, anastomosis of the right common carotid artery, and the right subclavian artery. Then the left common carotid artery and the left subclavian artery were reconstructed with the same branch of the quadrifurcated graft in an end-to-side and end-to-end manner, respectively. The postoperative course was uneventful in both cases. 


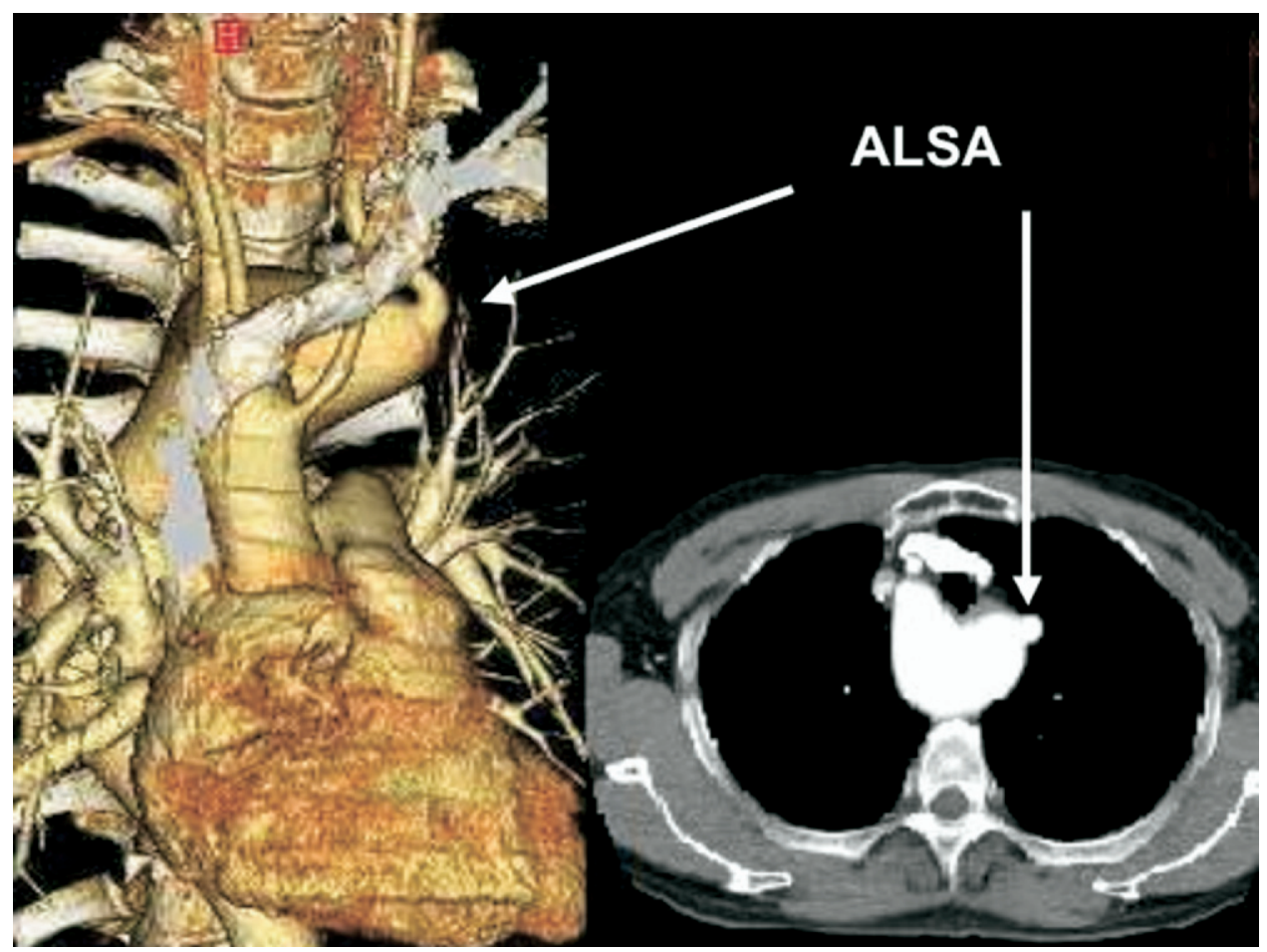

Figure 1. Preoperative multidimensional computed tomography. ALSA, Aberrant left subclavian artery.

\section{Discussion}

We have described the surgical technique of total arch replacement for a right-sided aortic arch aneurysm with an ALSA. We believe that hypothermic circulatory arrest with selective antegrade cere- bral perfusion, avoiding aortic crossclamping, is reliable for brain protection. One recent clinical study has shown that total arch replacement with deep hypothermic circulatory arrest (DHCA) using a quadrifurcated graft for a normal left-sided distal arch
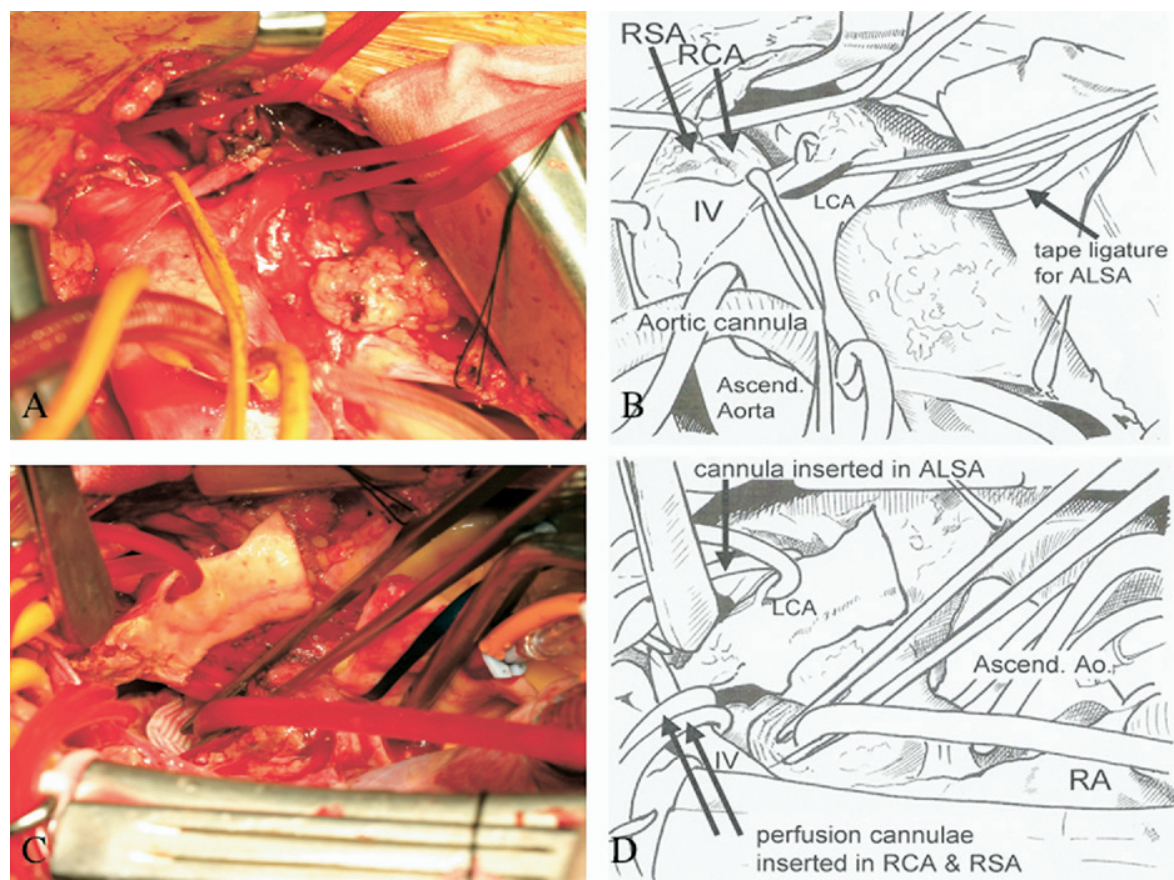

Figure 2. A, Cannulation and taping of the arch vessels. B, Schema of panel A. ALSA, Aberrant left subclavian artery; IV, innominate vein; $L C A$, left common carotid artery; $R C A$, right carotid artery; $R S A$, right subclavian artery. $\mathrm{C}$, Elephant trunk-type distal anastomosis. D, Schema of panel C. ALSA, Aberrant left subclavian artery; Ao, aorta; $R A$, right atrium; $L C A$, left common carotid artery; $R C A$, right carotid artery; $R S A$, right subclavian artery. 
aneurysm carries a low risk of perioperative stroke $(0.84 \%)$ compared with a standard left thoracotomy approach $(3.3 \%-8.1 \%){ }^{5}$ Manipulation of the aortic arch by using techniques such as crossclamping is thought to be a predisposing factor for stroke. In our patients a right thoracotomy with crossclamping of the aorta just distal to the right carotid artery was one possible surgical approach that could have been considered. However, we chose to perform total arch replacement through a midsternotomy with DHCA, and this was accomplished with no neurologic complications. Through a midsternotomy, it was easy to cannulate and reconstruct the arch vessels, including the ALSA. We completed the distal anastomosis by using an elephant trunk technique, even under the conditions of limited exposure provided by the midsternotomy. However, if the aneurysm had involved a large segment of the descending aorta or the patient had been very large, it would have been difficult to perform the distal anastomosis through a median sternotomy alone, and it might have been necessary to consider an additional right thoracotomy or two-stage operation (total arch replacement, followed by descending thoracic aorta replacement).

In conclusion, total arch replacement with DHCA through a midsternotomy alone is a useful approach for surgical repair of a right-sided aortic arch aneurysm with an ALSA in terms of brain protection and reconstruction. Surgical management for a normal left-sided distal arch aneurysm is applicable to a right-sided aortic arch aneurysm with an ALSA.

\section{References}

1. Knight L, Edwards JE. Right aortic arch. Types and associated cardiac anomalies. Circulation. 1974;50:1074-51.

2. Svensson LG, Crawfor DS. Congenital abnormalities of the aorta in adults. In: Svensson LG, Crawford DS, editors. Cardiovascular and vascular disease of the aorta. Philadelphia: WB Saunders; 1997. p. 153-74.

3. Tsukube T, Ataka K, Sakata M, Wakita N, Okita Y. Surgical treatment of an aneurysm in the right aortic arch with aberrant left subclavian artery. Ann Thorac Surg. 2001;71:1710-1.

4. Robinson BLAC, Nadolny EM, Entrup MH, Svensson LG. Management of right-sided aortic arch aneurysms. Ann Thorac Surg. 2001;72: $1764-5$.

5. Minatoya K, Ogino H, Matsuda H, Sasaki H, Yagihara T, Kitamura S. Surgical management of distal arch aneurysm: another approach with improved results. Ann Thorac Surg. 2006;81:1353-7.

\title{
Wound complications associated with the submammary incision for repair of congenital heart defects in female patients
}

\author{
Victor 0. Morell, MD, Peter D. Wearden, MD, PhD, and Mahesh S. Sharma, MD, Pittsburgh, Pa
}

T o improve the cosmetic result of the standard vertical median sternotomy incision, William and Hanlon ${ }^{1}$ in 1960 introduced the transverse submammary incision for median sternotomy. This operative approach has proved especially useful for female patients undergoing simple cardiac surgical procedures, resulting in a more esthetically pleasing scar. Although used in some centers, it has not gained worldwide popularity because of concerns related to potential wound complications, adequacy of surgical exposure, and access for reoperation. We have reviewed our experience with the submam-

From Children's Hospital of Pittsburgh, University of Pittsburgh, Pittsburgh, $\mathrm{Pa}$.

Received for publication July 31, 2006; accepted for publication Aug 7, 2006.

Address for reprints: Victor O. Morell, MD, Chief, Cardiothoracic Surgery, Children's Hospital of Pittsburgh, Associate Professor of Surgery, University of Pittsburgh, Room 2820, 3705 Fifth Ave, Pittsburgh, PA 15213 (E-mail: victor.morell@chp.edu).

J Thorac Cardiovasc Surg 2006;132:1475-6

$0022-5223 / \$ 32.00$

Copyright $\odot 2006$ by The American Association for Thoracic Surgery doi:10.1016/j.jtcvs.2006.08.004 mary incision in more than 100 patients undergoing repair of simple and complex congenital heart lesions.

\section{Patients and Methods}

This was a retrospective analysis of 106 consecutive female patients undergoing cardiac surgical procedures through the submammary approach for congenital heart defects. Their ages, surgical procedures, reoperations, and wound complications were examined.

A transverse surgical incision, extending from anterior axillary line to anterior axillary line, was performed at the level of the inferior mammary crease when present, or alternatively at the level of the xiphisternal joint in younger patients. The superior skin flap was created with electrocautery to the level of the sternal notch. Once the sternum was divided and the sternal retractor was placed, the skin flap was retracted superiorly with a self-retaining retractor. At the time of wound closure, a subcutaneous drain was placed under the superior skin flap in all cases.

\section{Results}

The mean patient age was 3.9 years (range 1 month -44 years). The surgical procedures, recorded in Table 1, included both simple and complex operations. There were no deaths in this group. Wound complications (Table 2) included seroma formation in 5 patients (4.5\%), superficial wound infection in 2 patients $(1.8 \%)$, hematoma formation in 1 patient $(0.9 \%)$, and partial superior skin flap 